\title{
El papel de las mujeres en la voz pública. ¿Cómo y dónde se genera la opinión en la Universidad?
}

\author{
The role of women in the public voice. How and where \\ is opinion generated in the University?
}

\section{Iolanda Piedra}

Presidenta Club XXIè Siegle

Presidenta de la Comisión Mujer y Empresa de Pimec y Miembro de su ejecutivo

Presidenta del Clúster Marítimo de Islas Baleares y de Cataluña

E-mail: ipiedra@pimec.net

\section{Las revoluciones feministas}

Históricamente se ha considerado la "superioridad" del hombre como natural. Ellos debían ejercer con responsabilidad el poder de la toma de decisiones políticas, económicas y sociales dejando a la mujer la crianza de hijos, cuidado de la casa y de su satisfacción sexual.

El concepto feminismo se refiere a la liberación de la mujer que al igual que otros movimientos han generado pensamiento y reflexión además de acción. El feminismo propugna eliminar las jerarquías y desigualdades entre sexos, pretende transformar las relaciones basadas en la asimetría y opresión sexual. Pero a lo largo de la historia puede verse como el feminismo no es homogéneo. La sensibilidad, oportunidad y la realidad del momento vivido por la mujer permitió emerger corrientes internas distintas.

En el siglo XVIII durante el Renacimiento, empieza a verse una toma de conciencia “colectiva" de esta situación, y podemos decir que nace el movimiento feminista de visión contemporánea. La mujer no podía estudiar, votar ni tomar decisiones familiares.

Durante la ilustración en Francia, nace el movimiento de la igualdad de clases sociales y la "Declaración de los derechos del hombre y del ciudadano" donde se excluyen los derechos de las mujeres. A través de su negación, ellas tomarán mayor consciencia del 
poco aprecio que se les tiene como seres que contribuyen con sus labores a la familia, economía y sociedad.

La primera ola del feminismo nacería con la Sra. Olympe de Gougues quien replicaría el texto anterior y desarrollaría la denominada "Declaración de los derechos de la mujer y de la ciudadana" donde revindicaría todos los derechos civiles para la mujer promulgando la igualdad jurídica y legal. En su obra afirmó que los "derechos naturales de la mujer están limitados por la tiranía del hombre, situación que debe ser reformada según las leyes de la naturaleza y la razón"

No es extraño que sus palabras le causasen la muerte y fuese guillotinada por el propio gobierno de Robespierre.

Estas mujeres como sus antepasadas no podían "pensar" ni ser "predictivas”, debían ser sumisas ante la autoridad, fuerza y "determinismo" que envolvía a los hombres. No hacía tantos siglos que las visionarias habían sido quemadas en la hoguera por brujas o ejecutadas por pitonisas al no atender a razonamientos donde es irrompible la cadena causa-efecto incluso en el ejercicio del "mal poder".

La acompañaría en época la Sra. Mary Wollistonecraft autora de "Vindicación de los derechos de la mujer" considerado el texto que funda el feminismo y rompe la idea básica del poder del hombre como natural que sustituye por cultural y transmitido por la educación. Su hija Mery Shelly escribiría la obra Frankenstein quien mezclaría ciencia y tecnología, la inmortalidad del hombre a modo de novela gótica.

La segunda ola del feminismo reconocido como "sufragismo", movimiento tanto intelectual como de acción social, nacería en los países anglosajones. En EEUU las mujeres que habían luchado por la independencia de su país se agruparían para la defensa de los esclavos y revindicarían esos derechos igualitarios en el Reino Unido donde se les limitaría su derecho a declarar en el Congreso Antiesclavista. Una audiencia con participación única de hombres no las aceptaría como voz pública por el hecho de ser mujeres. Asombradas ante unos hombres que les negaban su presencia física directa en tan respetable cámara, las Sras. Lucrecia Mott y E. Cady Stanton cambiarían el foco de su lucha ahora a favor de las mujeres y en (1848) en Seneca Falls expondrían la "Declaración de sentimientos" para recuperar los derechos civiles: igualdad de educación y de sufragio. 
A finales de la primera Guerra Mundial las mujeres verían recompensada su lucha pudiendo votar en Inglaterra lo que se extendería a Europa y América para la mujer "blanca". La burguesía feminista y blanca había conseguido alcanzar los derechos civiles básicos.

Pero la lucha continuaría visualizando una realidad, la diversidad de mujeres que existía en el mundo. La Sra. Sojourner Truth haría recordar que el resto de razas viven la doble exclusión en su caso por ser negra y mujer o la Sra. Flora Tristán, propulsora del llamado feminismo socialista recordaría que la mujer es la proletaria del proletariado, y daría luz a la doble exclusión por clase social y mujer.

La tercera ola del feminismo la podemos situar en 1949. En esa fecha la Sra. Simone de Beauvoir, escribiría el "Segundo sexo" nos ilustraría con su voz y escribiría la famosa frase "no se nace mujer se llega a serlo". En su obra enmarcaría los roles que se asocia a la mujer que la convierten en mujer. Beauvoir nos hablaría de androcentrismo lo que equivale a decir que "el hombre es la norma o medida de todas las cosas y la mujer es lo otro".

La Sra. Betty Friedan, socióloga nos instruiría con su bestseller "La mística de la feminidad" como la mujer puede sentirse insatisfecha si solo vive para cuidar de los demás. Friedan impulsaría el movimiento NOW (National Organitation for Women) iniciándose un feminismo liberal en los años 60, que pretende la reforma del sistema para obtener una igualdad entre ambos géneros. Uno de los principales objetivos para alcanzar la igualdad en el sistema, era garantizar la inclusión de la mujer en el mercado laboral.

En paralelo, el reconocimiento del patriarcado o poder del hombre en campos como: familiar, política, social, económica y científica, daría origen al llamado feminismo radical. Este nacería a partir de la idea de que aunque haya una legitimación de los derechos, el sistema sigue rigiéndose por unos valores sexistas, clasistas, racistas e imperialistas.

De hecho, dentro del feminismo de la época existen numerosos grupos con diversas tendencias y orientaciones, lo que supone hablar de "movimientos feministas" que podemos sintetizar en tres corrientes principales: una radical, otra socialista y otra liberal, entre cruzadas por las tendencias de la igualdad y la diferencia, véase por ejemplo 
Shulamith Firostene (1971), Delphy (1980) o Gomáriz (1991) entre otros autor/as si se desea profundizar en ello.

En los años 90 se tomará mayor conciencia de que no existe un único modelo de mujer sino múltiples que depende no solo de sus condiciones sociales o étnicas como las integradas en el colectivo LGTBI y se buscarán referentes para generar conciencia del rol y posición que debe tener la mujer en los distintos ámbitos de poder público vinculados a la empresa, la política y la universidad.

\section{2.- EI siglo XXI y el poder de la mujer en la política, la empresa y la universidad}

Estos tres ámbitos son en las últimas décadas motivo de análisis científico y de acción política para encontrar el equilibrio y restaurar la voz pública que históricamente se les ha arrebatado a las mujeres.

Aunque daré una pincelada en los tres para visualizar como estamos, pienso que vale la pena centrar la reflexión en el mundo universitario si entendemos esta "institución" como la transmisora de conocimientos al más elevado nivel y como motor del cambio. Personalmente estoy convencida que:

Los avances científicos y técnicos no deberían propiciar la perpetuación de las desigualdades y mucho menos hacer retroceder los derechos adquiridos por las mujeres.

Reflexionando sobre estos tres elementos de poder público a raíz de su evolución "parece", a la vista de algunos datos, que la universidad es el más lento.

El establecimiento de cuotas en los últimos años del siglo $\mathrm{XX}$ ha aumentado el número de parlamentarias en todo el mundo (Kumar y Sarangi), pero aún falta tiempo para que incremente realmente la presencia de la mujer en cargos de relevancia política (Teele).

Los datos del Centro para la Política y las mujeres Americanas (CAWP, de la Universidad de Nueva Jersey), muestran el bajo nivel de representación de las mujeres en las instituciones norteamericanas. Hay 23 en el Senado (el 23\% de los 100 escaños), 84 en la Cámara de Representantes (el 19\% de los 435 escaños), solo 6 gobernadoras (el 12\%), son el 25\% de los Legislativos estatales y el 20\% de los alcaldes de las 100 mayores ciudades. Pero la realidad es que la velocidad de crecimiento ha sido espectacular como apunta (O’Connor). 
La mujer también ha triplicado su representación en África (Tripp), son especialmente influyentes en América Latina (Pisiopo) y en Europa se ha alcanzado una máxima tras las últimas elecciones en 2019 con Ursula Gertrud von der Leyen al frente de la Comisión Europea o Christine Lagarde del Banco Central Europeo. Países como España, destacan por tener uno de los parlamentos con un acercamiento paritario mayor dentro de la UE.

Hay estudios que apuntan que las mujeres son más altruistas y cooperativas, tienen una elevada aversión al riesgo, su voluntad de comprobar los papeles a firmar son mayores y ello implica que pueden liberar la política de la cima de corrupción que viven algunos países como apunta (Kumar y Sarangi).

Es especialmente conmovedora al respecto la conferencia de Rosario Marín, tesorera de EEUU con el Presidente George Bush en 2001 titulada "Una líder entre dos mundos", intervención basada en su libro de igual nombre. O una visión contraria de cómo se puede ejercer el "mal poder" como apunta Lydia Cacho en su conferencia "Mujeres reinventando el poder" quien recuerda como “(...) el feminismo incorpora la filosofía de que es necesario contribuir al bienestar de unos y otros. El feminismo no es una batalla por el poder sino una tarea para reconstruir desde la reflexión personal y colectiva una nueva forma de ejercer el poder. Se necesitan hombres y mujeres por igual que desarrollen con empeño su trabajo, ejerzan su poder y contribuyan en la toma de decisiones".

Esta realidad se deteriora cuando hablamos de mercado de trabajo y poder por ejemplo en Europa o España. La Estrategia Europa 2020 pretende aumentar hasta un $75 \%$ la participación de la mujer en el mercado laboral tras demostrar que su incorporación al mundo laboral eleva el PIB.

El sistema de cuotas también está siendo adoptado en Europa para eliminar parte de las barreras que sufre la mujer en la empresa a la hora de acceder a los Consejos de Administración o a sus Comités de Dirección. El resultado si bien no es del todo deseable parece que su evolución es constante.

Y llegamos a una de las instituciones que debería generar mayor "opinión, reflexión y practicar la máxima de los términos democratización e igualdad”, la Universidad.

\section{3- ¿La universidad: uno de los últimos reductos de inmovilismo?}


El reconocimiento del éxito de las mujeres en el mundo de la investigación y la universidad nunca ha sido fácil.

La primera mujer en conseguir un Premio Nobel de física en 1903 fue Marie Curie. Pero su reconocimiento se limitó al de una colaboradora válida de su marido. Curie obtuvo su primer puesto de trabajo "remunerado" después de recibir ese galardón. Ocho años más tarde en 1911 recibiría el Premio Nobel de química. La única persona en ganar los dos en estas ciencias estuvo discriminada por el hecho de ser mujer. Hoy Curie es una de las pocas modelos que recuerdan los libros de texto y donde las jóvenes estudiantes pueden encontrar a una referente.

Es conocido que el número de mujeres con estudios universitarios ha crecido mucho en los últimos 50 años. Actualmente en muchos países desarrollados superan el 50\% de total de población matriculada aunque la distribución por carreras es desigual.

La presencia de mujeres en las áreas científicas como la física o las ingenierías es muy inferior a la de los hombres a diferencia de las ramas científicas biosanitarias y de las ciencias sociales, donde la proporción de estudiantes mujeres es mayor que la de los hombres como ocurre en España.

Y aquí las siguientes cuestiones entorno a -mujer y poder- en el mundo universitario: ¿Las mujeres son altavoces públicos científicos y de cambio? ¿Participan de forma paritaria en la toma de decisiones? ¿Lideran movimientos, líneas de estudios y expresan sus opiniones en la misma proporción e importancia que sus compañeros?

Resulta evidente que si la mujer no ha accedido en términos igualitarios en número a la universidad, como ocurre en la mayoría de países de cultura islámica, difícilmente las respuestas podrán ser positivas. Por ello, intentaré centrar la reflexión en Europa y España -donde no hay diferencias significativas por razón de sexo en el número de personas matriculadas en las universidades-, y además se trata de una realidad que conozco desde la vivencia y no sólo desde la investigación.

\section{La tasa de abandono}

A partir de los informes que publica de forma anual CSIC, se puede afirmar que la tasa de abandono de las mujeres que inician una carrera profesional universitaria es superior a la de los hombres. 
Analizando un período para el que se dispone de series 2001-2016. Las mujeres becarias en 2001 eran $981(57 \%)$ y los hombres $740(33 \%)$ para un intervalo de edad entre los 22 y 30 años. En 2016 el personal investigador funcionario menor de 45 años lo componían 152 mujeres (40\%) y 229 hombres (60\%).

Una simple regla aritmética pone en evidencia que el personal investigador funcionario menor de 45 años mujer en 2016 suponía sólo el 15,5\% del que fuera becario en 2001 mientras que para los hombres era del $31 \%$. En consecuencia la tasa de abandono es superior en la primera etapa de carrera profesional.

A partir de lograr la plaza de carácter funcionario deja de producirse ese abandono masivo por parte de las mujeres. De hecho, en 2001 el porcentaje de mujeres del cuerpo funcionarial investigador menor de 45 años era del 35,6\% mientras que en 2016 era de $36,24 \%$ cifra que se mantendría estable hasta los 65 años. Un estudio realizado por Loreal a 1300 personas investigadoras en el ámbito académico evidenciaría que las causas principales fueron: el sesgo de género (53\% Mujeres vs $2 \%$ Hombres), la falta de modelos $(28 \% \mathrm{M}$ vs $8 \% \mathrm{H})$, acceso a mentores $(34 \% \mathrm{M}$ vs $13 \% \mathrm{H})$ y falta de apoyo en el cuidado de los hijos (20\% M vs 7\% H).

\section{Sesgo y reconocimiento de méritos}

Analicemos de forma breve la principal causa: el sesgo de género. La falta de equilibrio paritario en las evaluaciones y procesos de selección es también un hecho en el mundo científico. Las mujeres científicas no solo sufren el efecto Mateo también el efecto Matilda. Ambos denotan la discriminación del desconocido y sin voz pública unido en éste caso, al hecho de ser mujer científica.

Efecto Mateo: Quítenle el talento para dárselo al que tiene diez, porque a quien tiene, se le dará y tendrá de más, pero al que no tiene, se le quitará aun lo que tiene. San Mateo en el evangelio, 25.

Efecto Matilda: La invisibilidad de la mujer en la ciencia es un fenómeno cotidiano. Fue identificado por la historiadora de la ciencia Margaret W. Rossiteren 1993, y denominado así en honor a Matilda Joslyn Gage, sufragista que denunció este fenómeno aún en pleno vigor en nuestros días. 
Una de las fuentes aún de referencia para España, es el Libro Blanco donde en su capítulo 3 se analiza las diferencias por género en la carrera científica. A modo de conclusión se describe como las mujeres publican menos que los hombres y dedican más horas a la docencia:

Las mujeres publican menos que los hombres en términos de artículos -aproximadamente 1,6 artículos menos- y dirigen menos tesis y tesinas -un $30 \%$ de mujeres ha dirigido alguna frente a un $40 \%$ de hombres-.

Los sesgos también existen en otros aspectos del mundo científico. Los méritos de un investigador/a dependen en gran medida del reconocimiento "de otros miembros de la comunidad científica" al trabajo realizado: aceptación en revistas del trabajo realizado, citas de los artículos publicados, invitaciones a conferencias, premios y nominaciones..., y algunos estudios evidencian ese efecto Matilda como tónica general.

El Consejo de Investigación Sueco en 1997 demostró que una mujer tenía que ser 2,5 veces más productiva que un hombre para recibir la misma calificación. En EEUU (2013) a partir del análisis de 5.483.841 artículos, se concluyó que todos aquellos suscritos por mujeres en posición dominante eran menos citados que los firmados por hombres en igual posición. Y recientemente, como cita la Iniciativa 11 de febrero (2019), otro grupo investigador ha estudiado 200.000 artículos del área de astronomía. Mediante un algoritmo de aprendizaje, como fórmula de análisis, se puso de manifiesto que si una mujer es primera autora tiene un $6 \%$ de menos citas y si se añade la calidad "aspectos científicos" el artículo debía haber sido citado un 4\% más lo que supone un sesgo estimado de género del $10 \%$. 


\section{La carrera hacia la cima un reto quimérico para la profesional universitaria}

Citando a (Hernández y Pérez) para la Universidad Española, "Paradójicamente, la Universidad sigue siendo de los ámbitos donde reina la desigualdad de género y donde el ascenso laboral de las mujeres es minoritario".

En España más de un $50 \%$ de las mujeres dedicadas a la docencia universitaria se concentran en los colectivos profesionales de profesores asociados y ayudantes de doctor (2018). Si analizamos la jerarquía organizativa, estas ocupan el $46 \%$ de los contratos de doctorandos, el $38 \%$ de las plazas de titular y el 25\% del cuerpo de catedráticas de las que solamente una de menos de 40 años ha llegado a ser catedrática.

Las que ocupan lugares de conducción y gestión en las estructuras jerárquicas de las universidades son muy pocas como se recogería en el Foro Mundial de Rectoras celebrado en abril del 2018 en China.

Si se tuviese que destacar algún rasgo de la tabla siguiente el número de ceros sería posiblemente lo que se resaltaría si se da un vistazo de forma rápida. Lamentablemente la tabla recoge el porcentaje de rectoras por país y por año según el Ranking Académico de las Universidades del Mundo cuyas siglas en inglés es ARWU.

\begin{tabular}{|c|c|c|c|c|c|c|c|c|c|c|c|c|c|c|c|c|}
\hline País & $\begin{array}{l}\text { Total de } \\
\text { rectores }\end{array}$ & $\%$ rectoras & 2003 & 2004 & 2005 & 2006 & 2007 & 2008 & 2009 & 2010 & 2011 & 2012 & 2013 & 2014 & 2015 & 2016 \\
\hline Estados Unidos & 1.379 & $17 \%$ & $16 \%$ & $14 \%$ & $15 \%$ & $13 \%$ & $16 \%$ & $16 \%$ & $18 \%$ & $19 \%$ & $20 \%$ & $20 \%$ & $19 \%$ & $17 \%$ & $17 \%$ & $16 \%$ \\
\hline Reino Unido & 237 & $17 \%$ & $23 \%$ & $17 \%$ & $17 \%$ & $21 \%$ & $15 \%$ & $13 \%$ & $19 \%$ & $17 \%$ & $17 \%$ & $11 \%$ & $15 \%$ & $14 \%$ & $18 \%$ & $25 \%$ \\
\hline Canadá & 214 & $20 \%$ & $25 \%$ & $27 \%$ & $27 \%$ & $36 \%$ & $21 \%$ & $23 \%$ & $13 \%$ & $18 \%$ & $13 \%$ & $13 \%$ & $18 \%$ & $19 \%$ & $13 \%$ & $13 \%$ \\
\hline China & 154 & $1 \%$ & 0 & 0 & 0 & 0 & 0 & 0 & 0 & 0 & 0 & 0 & 0 & 0 & 0 & $4 \%$ \\
\hline Alemania & 143 & $5 \%$ & 0 & 0 & 0 & 0 & 0 & 0 & 0 & $8 \%$ & $8 \%$ & $8 \%$ & $7 \%$ & $6 \%$ & $5 \%$ & $5 \%$ \\
\hline Japón & 121 & 0 & 0 & 0 & 0 & 0 & 0 & 0 & 0 & 0 & 0 & 0 & 0 & 0 & 0 & 0 \\
\hline España & 104 & $5 \%$ & 0 & 0 & 0 & 0 & 0 & 0 & $14 \%$ & $14 \%$ & $13 \%$ & $13 \%$ & 0 & 0 & 0 & $11 \%$ \\
\hline Corea del Sur & 100 & $3 \%$ & 0 & 0 & 0 & 0 & 0 & 0 & 0 & 0 & 0 & 0 & 0 & $14 \%$ & $11 \%$ & $13 \%$ \\
\hline Australia & 82 & $40 \%$ & & & & $50 \%$ & $67 \%$ & $33 \%$ & $33 \%$ & $33 \%$ & $38 \%$ & $30 \%$ & $30 \%$ & $50 \%$ & $46 \%$ & $40 \%$ \\
\hline Italia & 70 & 0 & 0 & 0 & 0 & 0 & 0 & 0 & 0 & 0 & 0 & 0 & 0 & 0 & 0 & 0 \\
\hline Israel & 49 & $22 \%$ & 0 & 0 & 0 & $25 \%$ & $25 \%$ & $25 \%$ & $25 \%$ & $25 \%$ & $25 \%$ & $25 \%$ & $25 \%$ & $25 \%$ & $25 \%$ & $33 \%$ \\
\hline Irlanda & 42 & 0 & 0 & 0 & 0 & 0 & 0 & 0 & 0 & 0 & 0 & 0 & 0 & 0 & 0 & 0 \\
\hline Taiwán & 41 & $2 \%$ & 0 & 0 & 0 & 0 & 0 & 0 & 0 & 0 & 0 & 0 & 0 & 0 & 0 & $20 \%$ \\
\hline Dinamarca & 38 & $5 \%$ & $50 \%$ & $50 \%$ & 0 & 0 & 0 & 0 & 0 & 0 & 0 & 0 & 0 & 0 & 0 & 0 \\
\hline Paises Bajos & 36 & $3 \%$ & & & & & & 0 & 0 & 0 & 0 & 0 & 0 & 0 & 0 & $14 \%$ \\
\hline Suecia & 33 & $45 \%$ & 0 & 0 & 0 & 0 & $100 \%$ & $100 \%$ & $100 \%$ & $100 \%$ & $100 \%$ & $50 \%$ & $67 \%$ & $50,00 \%$ & $33 \%$ & $38 \%$ \\
\hline Suiza & 31 & $13 \%$ & 0 & 0 & 0 & 0 & 0 & 0 & 0 & 0 & 0 & 0 & 0 & 0 & $25 \%$ & $50 \%$ \\
\hline Sudáfrica & 28 & 0 & 0 & 0 & 0 & 0 & 0 & 0 & 0 & 0 & 0 & 0 & 0 & 0 & 0 & 0 \\
\hline Austria & 26 & $42 \%$ & & & & & & & & & $50 \%$ & $50 \%$ & $50 \%$ & $50 \%$ & $40 \%$ & $20 \%$ \\
\hline Brasil & 25 & 0 & 0 & 0 & 0 & 0 & 0 & 0 & 0 & 0 & 0 & 0 & 0 & 0 & 0 & 0 \\
\hline Francia & 24 & $29 \%$ & & 0 & 0 & 0 & 0 & 0 & 0 & $50 \%$ & $100 \%$ & $100 \%$ & $100 \%$ & $33 \%$ & $25 \%$ & $17 \%$ \\
\hline Rusia & 24 & 0 & 0 & 0 & 0 & 0 & 0 & 0 & 0 & 0 & 0 & 0 & 0 & 0 & 0 & 0 \\
\hline Finlandia & 22 & 0 & 0 & 0 & 0 & 0 & 0 & 0 & 0 & 0 & 0 & 0 & 0 & 0 & 0 & 0 \\
\hline
\end{tabular}

Fuente: ARWU

La preocupación real es el estancamiento e incluso la evolución negativa del porcentaje de mujeres que llegan a rectoras. 
A raíz de estas evidencias la respuesta a las tres preguntas que realizaba al inicio de este tercer apartado es la misma: no, no y no. La lucha por la igualdad de género, la promoción profesional y, por tanto, la voz pública de las científicas y docentes universitarias es urgente.

Posiblemente, las mujeres debemos activar una cuarta ola de feminismo activo $\mathbf{y}$ colectivo, para evitar un retroceso en el camino recorrido.

\section{Bibliografía}

Castells, C. (comp.) (1996). Perspectivas feministas en teoría política. Paidós, Barcelona.

Gomáriz, E. (1992). "Los estudios de género y sus fuentes epistemológicas: periodización y perspectivas" en AAVV, Fin de Siglo- Género y cambio civilizatorio, Santiago, Isis Internacional- Ed. de las Mujeres Na 17.

De Gouges, O. (1989). "Los derechos de la mujer y la ciudadana”. En Alonso, I. y Belinchon, M. (Eds.): 1789-1783. La voz de las mujeres en la Revolución Francesa. Cuadernos de Quejas y otros textos. Barcelona: La Sal Ediciones de les Dones (pp. 132$133)$.

Dawn Lagan Teele (2019). Un siglo de mujeres en la política.

Hernández Armentero, J. y Pérez García, J. A. (2017). Demografía universitaria española: aproximación a su dimensión estructura y evolución. Studia XXI. Fundación Europea Sociedad y Educación.

O’Connor, K. (2019). La creciente importancia de las mujeres para el Partido Demócrata. Chandan Kumar Jha y Sudipta Sarangi. ¿Reduce la corrupción la elección de más mujeres políticas?

Loreal y Cel Associates. Barriers for women scientistis. Survey Report.

Ministerio de Ciencia e Innovación - UMC. Libro blanco. Situación de las Mujeres en la Ciencia Española. Cap 3. Diferencias por género en la carrera científica. Evidencia empírica.

Nicholson, L. (comp.) (1994): Feminismo/posmodernismo, Feminaria Editora, Buenos Aires. 
Iniciativa ciudadana 11 de Febrero (2019). Artículo. Sesgo en la evaluación y en el reconocimiento de méritos.

Wollstonecraft, M. (1996). Vindicación de los derechos de la mujer. Madrid: Cátedra.

Información estadística:

Universidad española en cifras. Conferencia de Rectores de las Universidades Española (CRUE).

Sistema integra de Información universitaria (SIIU). Ministerio de Educación.

Por internet:

Evangelio según San Mateo. Mateo 25: 14-30, en La parábola de los talentos

http://www.bantaba.ehu.es/formarse/ficheros/view/Historia_del_Movimiento_feminista. pdf?revision_id=53767\&package_id=33304

Lydia Cacho - "Mujeres reinventando el poder"

https://www.youtube.com/watch?v=FuEyVhcJstA

Rosario Marín. Ponencia en Forbes foro mujeres poderosas RD- "Una líder entre dos mundos"

https://www.youtube.com/watch?v=IkGAFMwtLdw

Historia del movimiento feminista.

http://www.bantaba.ehu.es/formarse/ficheros/view/Historia_del_Movimiento_feminista. pdf?revision_id=53767\&package_id=33304

Por qué las chicas no quieren ser ingenieras.

https://www.elmundo.es/espana/2015/07/27/55b025f7268e3e3b6e8b459b.html?platfor $\mathrm{m}=$ hootsuite

Studia XXI, cuaderno de trabajo 9. Fundación Europea Sociedad y Educación. https://issuu.com/efse/docs/ct9-studia-xxi 